\title{
Residual Stress Measurement of Shot-Peened Steel Rings by Barkhausen Noise, ESPI Hole-Drilling and X-Ray Diffraction
}

\author{
Theo J. Rickert ${ }^{1, a^{*}}$, James J. Thomas ${ }^{1, b}$ and Lasse Suominen ${ }^{2, c}$ \\ ${ }^{1}$ American Stress Technologies, Inc., 540 Alpha Drive, Pittsburgh PA 15238, USA \\ ${ }^{2}$ Stresstech Oy, Tikkutehtaantie 1, 40800 Vaajakoski, Finland \\ aTheo.Rickert@astresstech.com, bJames.Thomas@astresstech.com, \\ cLasse.Suominen@stresstech.com \\ * corresponding author
}

Keywords: Residual Stress Measurement, X-Ray Diffraction, ESPI, Hole-Drilling, Barkhausen Noise, Quality Control, Shot-Peening

\begin{abstract}
Shot-Peening is used to generate surface-near compressive residual stresses in final shape parts, usually to improve their fatigue properties. The success of the process can be checked in different ways. XRD and Hole-Drilling determine quantitative stress depth profiles in specific locations. Barkhausen noise measurements evaluate a near-surface zone in real-time and can cover large areas quickly. It is a fully nondestructive method. This study compares ESPI Hole-Drilling, which is a very fast technique, and XRD, which is very precise but slower, to Barkhausen Noise results for the case of three steel rings shot-peened with different intensities.
\end{abstract}

\section{Introduction}

Residual stress can be measured using many different techniques. All have specific applications where they excel. Experimental comparisons of the techniques can illustrate the differences and point out advantages and disadvantages of the different techniques. This study used three steel rings that were shot-peened differently.

$\mathrm{X}$-ray diffraction (XRD) stress measurement is commonly used for steels and many other materials that possess crystalline structure. Hole-drilling is the other most frequently used method for stress measurement and does not require crystalline microstructure. But it assumes that the material around the hole behaves homogeneously, like an isotropic, single phase. While the holedrilling technique is traditionally performed using strain-gages to measure the material response, the method employed here uses laser light. Electronic speckle pattern interferometry (ESPI) combined with digital photography allows measuring tiny shifts of the surfacearea around the hole due to the hole-drilling and stress re-balancing. These surface displacements are measured as a fraction of the laser wavelength. No calibration is required. The third method requires that the material is ferromagnetic. It measures the Barkhausen noise $(\mathrm{BN})$ signal originating from a near-surface zone of a sample in the presence of a changing magnetic field. Due to its nature as a relative measure, which is typical in non-destructive methods, BN is generally not used to quantify residual stress but rather for process or quality control, identifying locations on parts that have different properties / microstructures, including due to local residual stresses. Measurement results are displayed immediately - while the sensor moves across the part surface. It is a fully non-destructive method and interrogates a depth range on the order of $0.1 \mathrm{~mm}$. In comparison, a non-destructive measurement by XRD captures information from a much shallower zone, maybe one tenth the thickness, that is very sensitive to scratching and other surface artifacts. Residual stress depth profiles by XRD are at least as destructive as hole-drilling since the material volume removed is larger. 


\section{Experimental}

The three samples are shaped like sections of heavy tube or tall rings. They are approximately 4" $(100 \mathrm{~mm})$ in diameter (outer), $1 / 2$ " $(13 \mathrm{~mm})$ thick, are made of HSLA steel 4340M and shot peened on the outer diameter. The shot-peening intensity of the three rings differs because different air pressures were used. The Almen intensities ranged from 15.7 to 9.8 and 5.9 for Rings A thru C, respectively. The coverage was the same and the visual appearance is identical.

X-Ray Diffraction. The X-ray diffraction measurements were made with an XStress 3000 system, a portable instrument dedicated to residual stress measurement with minimal restrictions on sample size and shape. The measurement geometry is modified- $\chi$, with two position-sensitive detectors whose peak position data is averaged. The tilt range was $\pm 45^{\circ}$; the radiation source had a Cr-target; the diffraction peak analyzed was ferrite $\{211\}$. The material removal was accomplished by electro-polishing. The depths were measured with a dial indicator, part of a depth measurement station where the mounted part was positioned the same way after every electro-polishing step.

ESPI Hole-Drilling. The ESPI hole-drilling measurements were made with a Prism ${ }^{\circledR}{ }^{1}$ system, which uses an electric drill and a green laser $(532 \mathrm{~nm})$. The hole diameter was $1 / 32$ " $(0.8 \mathrm{~mm})$ and the rotational speed 30,000 rpm. Depth determination relied on the linear stage on which the drill was mounted, i.e. the position the actuator reports. The samples were positioned for measurement in hoop direction (full sensitivity). The depth increments ranged from $\sim 0.01 \mathrm{~mm}$ at the lowest depths to $\sim 0.05 \mathrm{~mm}$ at the highest.

The measurement process with the ESPI hole-drilling instrument differs somewhat from straingage hole-drilling. The optical measurement is sensitive to vibrations; it is performed on an optical table or breadboard. The sample is clamped in a manner appropriate for part shape and measurement location and positioned in front of the drill for perpendicular drilling. Illumination stand and camera are oriented to focus on the drilling location. Since the measurement surfaces are reflective the samples were lightly spray-painted prior to measurement to improve the optical properties. The last preparatory step is to identify the position of the stage where the drilling tool first contacts the part: zero-depth. The stress calculation requires ESPI images of the measurement surface before and after each drilling increment. Four images describe each condition. This allows the determination of surface displacements as a fraction of the wavelength. Thus, at least one image set at zero-depth and one for each depth increment are necessary.

The stress calculation algorithm uses the Integral Method [1] and incremental computation [2]. The same Tikhonov regularization was used in all cases, which links the stress values at different depths and leads to curve smoothing. The stress calculation algorithm is compatible with the requirements described in the strain-gage hole-drilling ASTM standard [3].

Barkhausen Noise Measurements. Barkhausen noise (BN) measurements were completed with a Stresstech Microscan 600 central processing unit and a High Power OD sensor magnetizing in the circumferential direction. The sample was magnetized at a frequency of $125 \mathrm{~Hz}$ with a voltage of 6.0 Volts peak-to-peak. The Barkhausen noise signals were measured within a frequency range of $70-200 \mathrm{kHz}$ using bandpass filtering with a sampling frequency of $2.5 \mathrm{MHz}$. The result of these measurement parameters is an approximate effective measurement depth of 25-50 $\mu \mathrm{m}$ [4].

Each sample was measured by clamping the sensor onto the sample. Twenty BN bursts were collected (10 magnetizing periods) and averaged for each measurement point using the Microscan software. The root-mean-square (RMS) of the BN signal over the measurement time is calculated and reported in $\mathrm{mV}$.

The BN RMS signal is sensitive to relative changes in stress in ferromagnetic materials and increases with higher levels of stress (less compressive, more tensile) in iron-based alloys. Magnetization must be oriented parallel to the axis of desired stress measurement as the magnetostrictive effect is proportional to $\cos ^{2} \theta$ where $\theta$ is the angle between the stress and the applied magnetizing field.

\footnotetext{
${ }^{1}$ Prism is a registered trademark of American Stress Technologies, www.astresstech.com.
} 


\section{Results}

X-Ray Diffraction. The XRD depth profiles were made in three directions, hoop, axial and $45^{\circ}$ in-between. The results for each ring were basically identical (Fig. 1). However, the three shotpeening treatments generated distinctly different stress profiles in the three rings (Fig. 2). According to the XRD results, the depth affected by shot-peening varies by about a factor of two while the maximum compressive stresses are similar. Interestingly, the surface stresses show an inverse correlation with the shot-peening depth. The diffraction peak widths change from about $5^{\circ}$ FWHM at maximum compressive stresses to over $6^{\circ}$ at the highest depths measured. - XRD error bars are plotted in Figure 3 but hardly noticeable since they are very small on this scale.

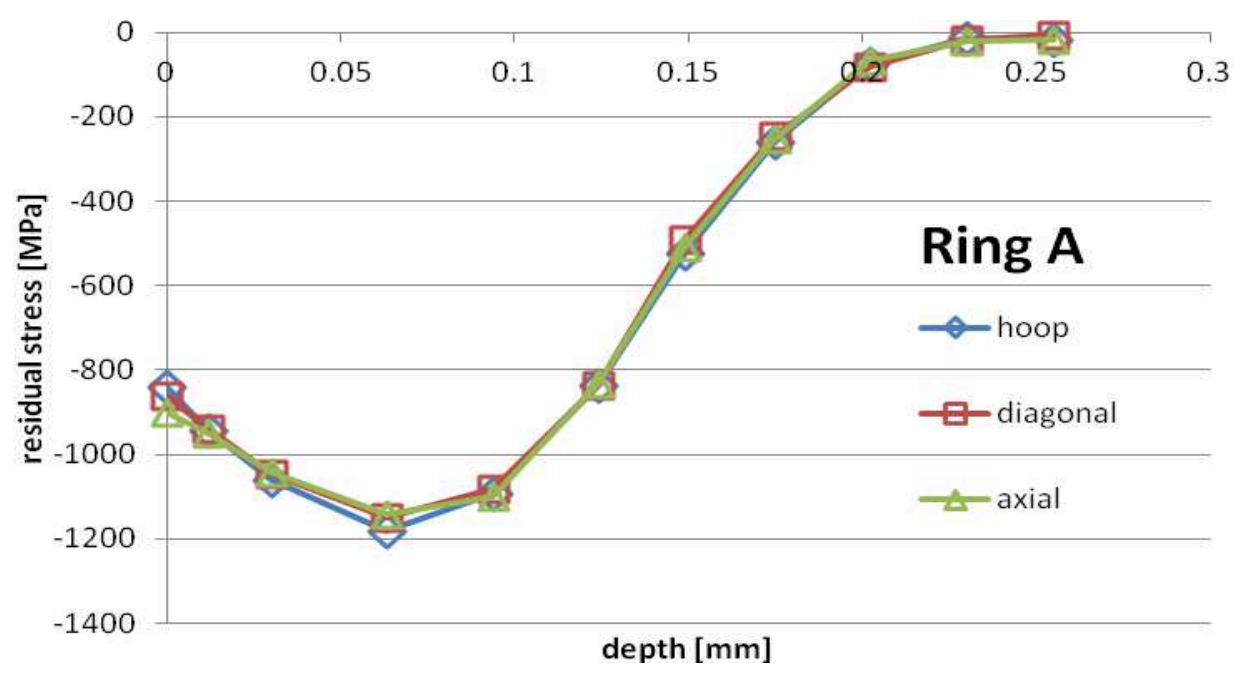

Fig. 1: The XRD residual stress depth profiles for the three directions measured are basically identical in all three rings.

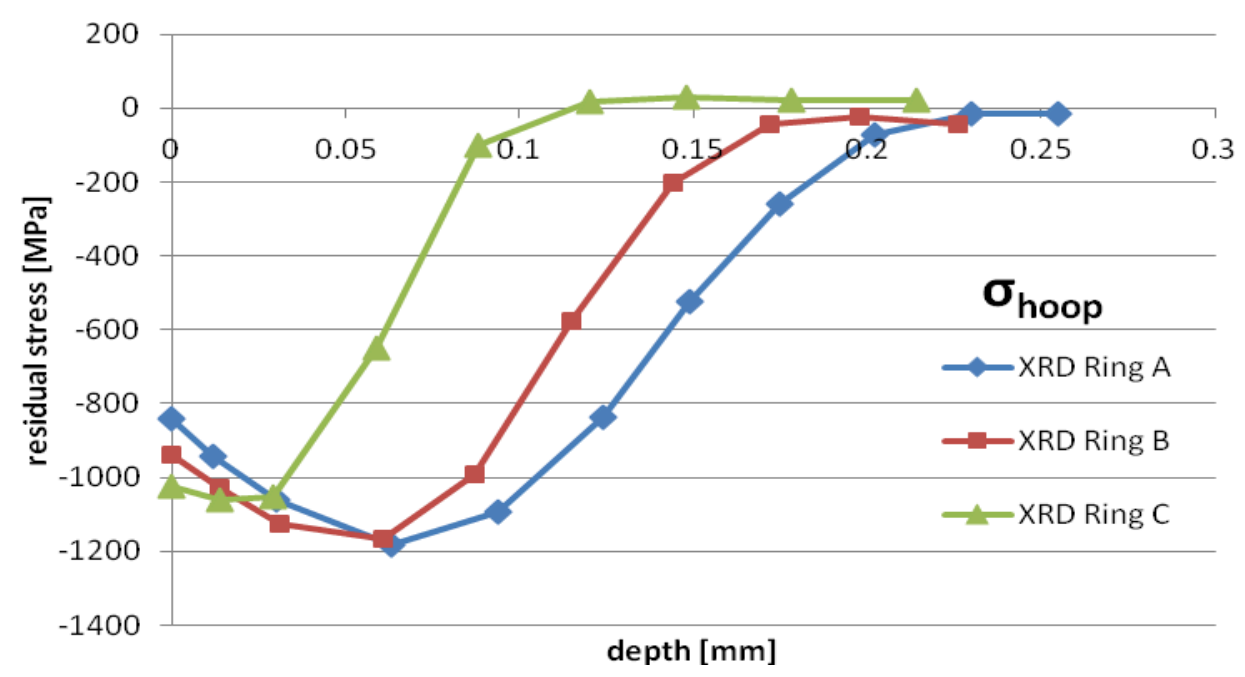

Fig. 2: The depth zone affected by shot-peening differs significantly in the three rings (XRD) while the maximum compressive stresses are comparable. The surface and near-surface stresses are distinctly different.

ESPI Hole-Drilling. Three holes were drilled in each ring to allow an estimation of measurement repeatability. The results are very similar (Fig. 3). The stress depth profiles also match the XRD results quite well - for hoop direction as well as axial direction. The ESPI data tend to underestimate the stresses at low depths. The depth profiles within each triplet are slightly shifted against each other. 

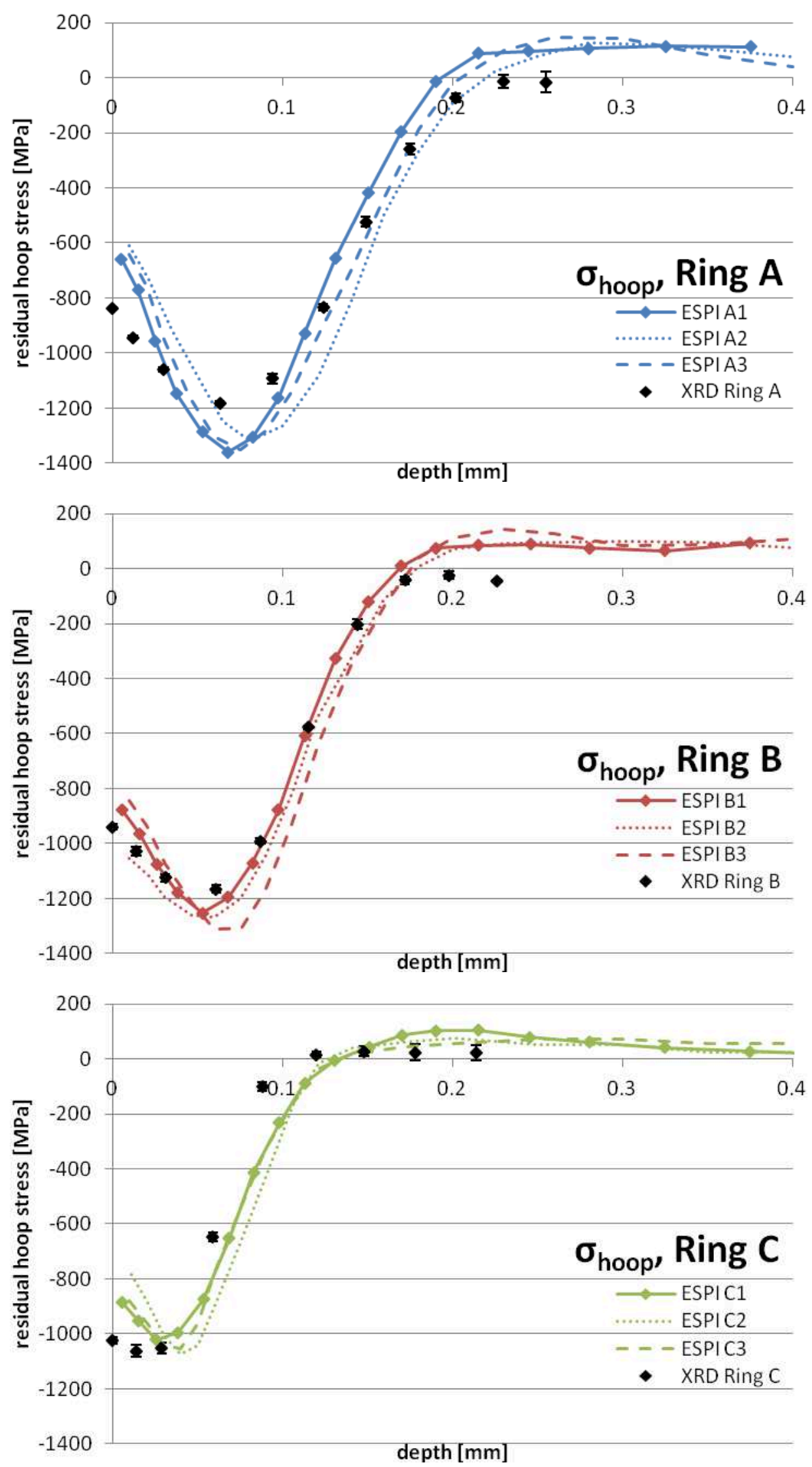

Fig. 3: The results of the three ESPI measurements per ring are quite similar and agree well with the XRD data. Note the error bars with the XRD data.- Note also that the hole-drilling data were plotted at the mid positions of the respective depth increments for this comparison (not standard for hole-drilling data). 
Barkhausen Noise. Rectified BN bursts are illustrated in Figure 4. The results for positive and negative bursts are basically mirror images of each other. The three rings are well separated. Higher signal strength indicates less compressive stresses. This correlates with the very first section of the stress depth profiles (Fig. 2).

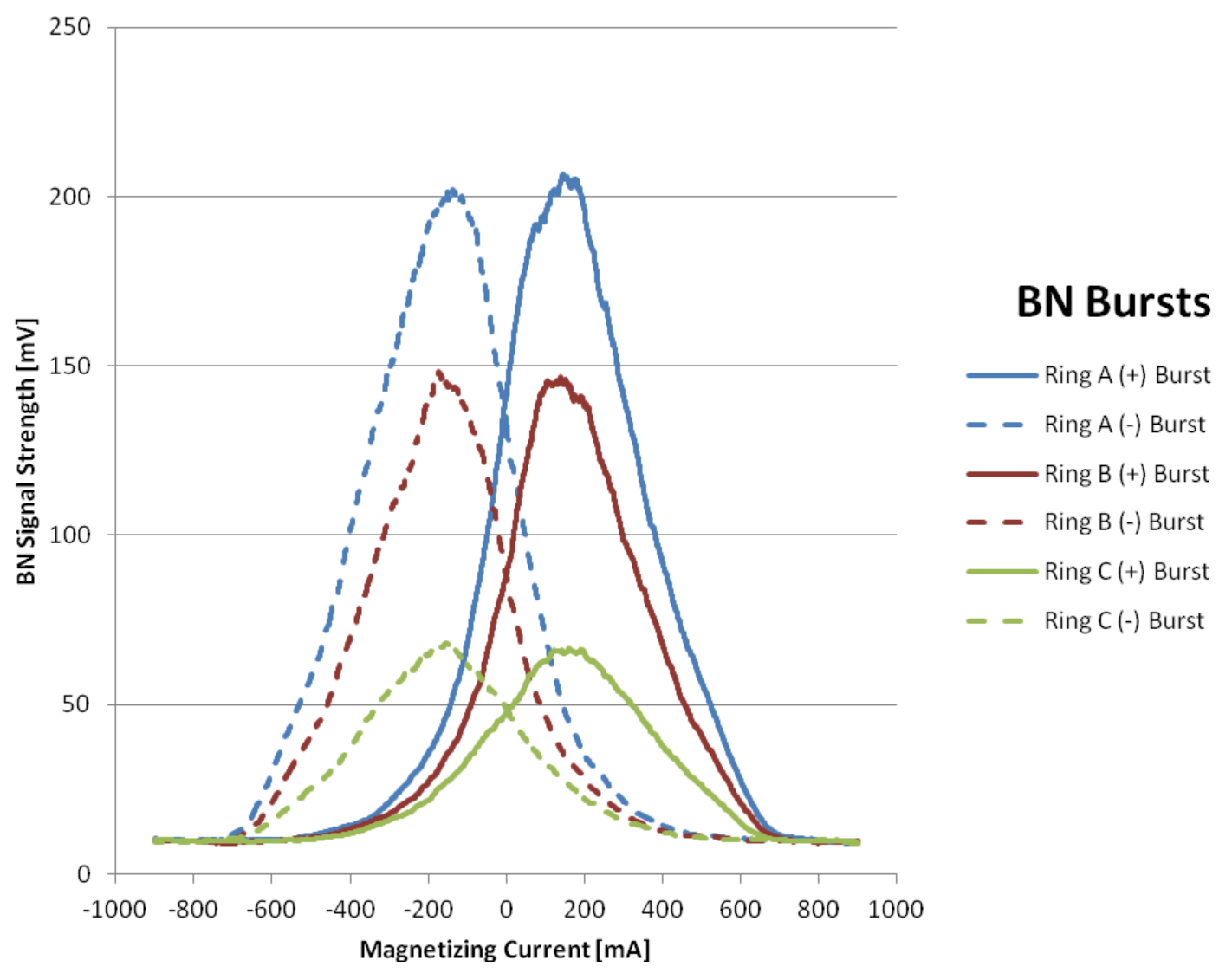

Fig. 4: Rectified BN bursts, including both positive (+) and negative (-) bursts, are charted as a function of magnetizing current. Positive and negative bursts correspond with opposite magnetizing directions in an alternating field.

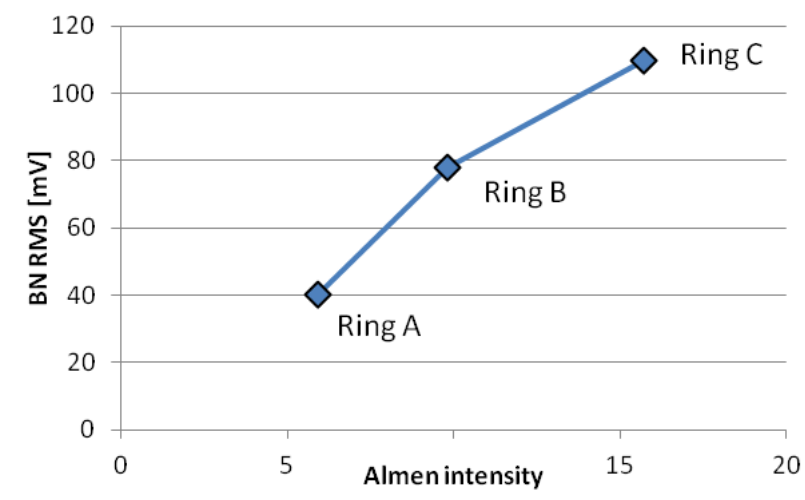

Fig. 5: $\quad$ BN RMS as a function of Almen intensity of the shot-peening process.

\section{Discussion}

XRD and ESPI. The shot-peening process was very non-directional, as evidenced by the similarity of the XRD data in the three measurement directions and confirmed by the ESPI measurements. The differences in the stress profiles between the three shot-peening treatments were resolved readily by both depth profiling methods. Given the similarity of the results, the ESPI 
method has an advantage over XRD in this case in that it is much faster. But just like the quality of XRD results depends on the quality of the diffraction peak, the hole-drilling method depends on the quality of the hole drilled. Measurement quality will likely change differently for the two methods when material or microstructure is different.

One difference between these two methods is that the data gathered by XRD originates from a relatively thin layer while hole-drilling analyzes the changes occurring for each complete drilling increment. Thus, it is particularly important for the hole-drilling method to choose small depth increments for describing sharp stress gradients. However, a smaller depth increment means that the measurement signal is smaller and better sensitivity is required. Regularization during stress calculation is important to smooth data scatter. The hole-drilling data at low depths are particularly strongly affected by regularization because the volumes of removed material are especially small.

The shifts observed for the ESPI stress curves for the same sample occur in depth direction. They are likely to some degree caused by differences in zero-depth determination. While this error can generally be controlled by increased attention to zero-depth determination, the significant surface roughness of the samples makes it difficult to decide what cutting pattern indicates zerodepth. Some visible curve shift has to be expected between multiple measurements.

Barkhausen Noise. The Barkhausen noise measurements were made in the typical fashion, with the main emphasis on magnifying the differences between the samples at hand. The differences found correlate well with the very first part of the stress depth profiles. In this depth range, the best comparison is with the XRD data since hole-drilling has higher relative errors at low depths, e.g. zero-depth accuracy and surface roughness. To increase depth range with BN measurements, one can use lower frequencies. But the measurement will always include a stronger signal from lower depth so that smaller differences near the surface can mask bigger differences at depth that have the opposite effect on Barkhausen noise. For the current samples the use of lower frequencies would reduce the differences between the samples. The strength of the Barkhausen Noise Method is in identifying relative differences between samples within a group or between locations and surfaces on a single sample.

Measurement Error. The XRD stress calculation generates standard deviation values. In this case they were very small relative to the stress values owing to the good measurability of steels and the large stresses. The hole-drilling stress calculation doesn't include any measurement error quantification. The three repeat measurements indicate significant variability compared to the XRD data.

\section{Acknowledgement}

The authors thank Mr. Willem Hamer of KLM for providing the samples and the permission to publish this data.

\section{References}

[1] G. S. Schajer and M. Steinzig, Full-Field Calculation of Hole-Drilling Residual Stresses from ESPI Data, Experimental Mechanics, Vol.45, No.6, pp.526-532, 2005

[2] G.S. Schajer and T.J. Rickert, Incremental Computation Technique for Residual Stress Calculations Using the Integral Method, Experimental Mechanics, Vol.51, No.7, pp.1217-1222, 2011

[3] Standard Test Method for Determining Residual Stresses by the Hole-Drilling Strain-Gage Method, ASTM E837-08e2

[4] S. Tiitto and S. Saynajakangas, Spectral damping in Barkhausen noise, IEEE Transactions on Magnetics, Volume 11, Issue 6, pp. 1666-1672, 1975 